\title{
RELACIONES ENTRE RENDIMIENTO E INTELIGENCIA EMOCIONAL EN SECUNDARIA
}

\author{
Relations between performance and emotional inteligence in secondary \\ education
}

\author{
Federico Pulido Acosta \\ e-mail: feanor_fede@hotmail.com \\ Francisco Herrera Clavero \\ e-mail: fherrera@ugr.es \\ Universidad de Granada. España
}

\section{RESUMEN}

Este trabajo tiene como objetivo analizar los tipos y niveles de Rendimiento Académico e Inteligencia Emocional, en función de la edad, género, cultura y estrato socioeconómico, así como contemplar las relaciones entre ambas variables. Para ello, se ha contado con 811 participantes. Considerando la cultura, el grupo mayoritario es el de religión musulmana (71.6\%), siendo el $46.1 \%$ varones y el resto de la muestra (53.9\%) mujeres. Como instrumentos de evaluación se han empleado un cuestionario elaborado para evaluar la $\mathrm{E}$ y las calificaciones de los alumnos. En IE aparecen niveles medio-altos, influyendo la edad, el género, la cultura/religión y el estatus. Aparecen niveles medios en RA, influyendo edad, cultura y estatus. Se halló relación entre RA e IE, siendo directamente proporcionales.

PALABRAS CLAVE: emociones; estados emocionales; rendimiento académico; inteligencia emocional; contexto pluricultural.

\section{ABSTRACT}

This work has as main objective which in to analyze the types and levels of Academic Achievement and Emotional Intelligence, according to age, gender, religion and socioeconomic and cultural factor, as well as the links between both variables. To make that possible we focused on 811 participants; $71.6 \%$ of the total were Muslims and $28.4 \%$ Roman Catholic, $46.1 \%$ were male and $53.9 \%$ female. The techniques used in this survey were an El questionnaire, as well as the students' grades. The results show medium-high levels of El. This is influenced by age, gender, culture and socioeconomic and cultural factor. We found medium levels of AA. This is influenced by age, culture and socioeconomic and cultural factor. There is a statistically significant relationship between AA and El. This relationship is directly proportional.

KEY WORDS: emotions; emotional states; academic achievement; emotional intelligence; pluricultural context.

Cómo referenciar este artículo / How to reference this article:

Pulido Acosta, F. \& Herrera Clavero, F. (2018). Relaciones entre rendimiento e inteligencia emocional en secundaria. Tendencias Pedagógicas, 31, 165-186. doi: http://dx.doi.org/10.15366/tp2018.31.010 


\section{INTRODUCCIÓN}

No se deben obviar las emociones y resulta fundamental en la vida saber reaccionar de manera adecuada ante ellas, saber gestionarlas. De esta manera, una realidad que tradicionalmente se ha venido considerando como un elemento que podía llegar a hacer a las personas más débiles, se convierte en un arma muy importante con la que afrontar la vida, estando relacionada con el éxito personal, académico y profesional.

Resulta importante comenzar discriminando entre aquellos trabajos que consideran la inteligencia emocional como conjunto de habilidades de aquellos que la tienen en cuenta de una manera autopercibida. El tipo de cuestionario que se pretende emplear, se acerca a esta última concepción de inteligencia emocional, lo que permite que se pueda hablar de un conjunto de habilidades específicas que se convierten en uno de los principales predictores de la adaptación emocional ajustada (Pulido y Herrera, 2016).

Resulta fundamental tener en cuenta todos los elementos que pueden llegar a depender de las capacidades relacionadas con la inteligencia emocional. En este sentido, elementos integrados en este conjunto de habilidades aparecen como predictores de una mayor calidad de vida, mayor salud mental, bienestar personal y laboral, una adaptación emocional más ajustada, mejor ajuste psicológico e incluso mejor capacidad para la resolución de problemas sociales (Azpiazu, Esnaola, \& Sarasa, 2015 y Cejudo, 2016).

Se ha de destacar que el proceso de desarrollo emocional provoca importantes cambios en todas las capacidades que forman parte de la inteligencia emocional, desarrollándose con el repertorio emocional del sujeto. Esta realidad es otra forma de contemplar la incidencia que tiene la edad en las puntuaciones de inteligencia emocional (Gomes y Pereira, 2014). Las habilidades emocionales han estado tradicionalmente ligadas al género femenino, siendo las mujeres las que presentan niveles superiores (Azpiazu et al., 2015; Pulido y Herrera, 2016; Caballero-Dominguez, Suárez-Colorado y Brugesa-Carbono, 2015 y Cejudo, 2016), lo que no hace más que aumentar el estereotipo que indica que las mujeres son más "emocionales" que los varones. La cultura también es un elemento a considerar, si se tienen en cuenta las diferencias existentes en los niveles de inteligencia emocional en función del grupo cultural (Oberle, Schonert-Reichl y Zumbo, 2011 y Pulido y Herrera, 2016), dado que la cultura trae consigo numerosas diferencias en los patrones educativos y los valores transmitidos, elementos que se relacionan con el desempeño emocional. En relación con lo comentado, el estatus socioeconómico y cultural también es determinante en los niveles reflejados en esta variable (Pulido y Herrera, 2016), presentando mayores capacidades emocionales aquellas personas pertenecientes a estatus superiores.

En segundo lugar, aparece el Rendimiento Académico. Desde esta perspectiva, se introduce nuestra concepción personal sobre el concepto, que se refiere al nivel de conocimientos y destrezas escolares exhibidos por los estudiantes. En este caso, se van a emplear las calificaciones escolares como indicativo de éste. Por esto, se ha de considerar a Gómez-Castro (1986, p. 269), ya que "al comparar los resultados de las calificaciones objetivas a través de una prueba estandarizada y las que otorgan los profesores, indica cierta 
asociación entre ellas. Todas las correlaciones obtenidas entre las distintas subpruebas y las calificaciones son estadísticamente significativas ( $p>01)$.

Pulido y Herrera (2015) establecen diferencias en función del género, que actúa como predictor del rendimiento. En este trabajo fueron las mujeres las que presentan mejores resultados que los varones. Sin embargo, la cultura es un factor mucho más determinante en las calificaciones académicas. De nuevo, Pulido y Herrera (2015) demostraron diferencias en los niveles, en dos muestras de cultural diferentes, no sólo en el rendimiento académico general, sino también en cada una de las materias. En este sentido, se mencionan las dificultades para la integración socio educativa (Oropesa, Moreno, Pérez \& Muñoz-Tinoco, 2014) y las diferencias de estatus socioeconómico y cultural (Cervini, Dari y Quiroz, 2014) como importantes justificantes de estas diferencias significativas. Por otro lado, en el contexto pluricultural en el que se pretende desarrollar el estudio, tiene mucha importancia las importantes diferencias en cuanto a la lengua materna entre musulmanes y cristianos, tal como informan algunos autores (Roa, 2006) del mismo modo que ocurre en otros entornos en los que aparece esta diversidad lingüística (Siqués y Vila, 2014). Otro de los elementos considerados como determinantes (Pulido y Herrera, 2015) es el estatus, que guarda una estrecha relación con el grupo cultural de pertenencia. Como justificante se puede volver a mencionar lo comentado en el trabajo, ya mencionado, de Alonso y Román (2014).

Las competencias emocionales son fundamentales para la mejora del rendimiento académico y la prevención de la conflictividad (Gorostiaga y Balluerka, 2014). Esta realidad se repite en otros muchos trabajos, en los que se demuestra una relación directamente proporcional entre las puntuaciones en inteligencia emocional y el rendimiento académico, establecido por la calificación media del sujeto (Codier y Odell, 2014; Hanin \& Van Nieuwenhoven, 2016; Pulido y Herrera, 2015). Dichas habilidades pueden actuar como factor de vulnerabilidad, influyendo negativamente en los estudiantes, no sólo dentro, sino también fuera de las situaciones escolares, influyendo sus carencias en el rendimiento académico, dado que estas habilidades están íntimamente relacionadas con el desempeño académico (Gutiérrez \& Expósito, 2015). De esta forma, la emocionalidad es fundamental para llegar a un alto rendimiento, no sólo a nivel académico, sino también social, empresarial, personal... Para realizar aprendizajes significativos es esencial el parámetro asociado a la transformación del campo emocional (Pacheco, Villagrán \& Guzmán, 2015). Sin embargo, el desarrollo de este tipo de habilidades no se encuentran atendidas de forma adecuada en la actual currículo de nuestro país (Aguaded y Pantoja, 2015). Por lo que se vuelve a incidir en la importancia de considerar y potenciar las competencias emocionales para mejorar el proceso de aprendizaje del alumnado (De Haro y Castejón, 2014 y Di Giusto, Martín, Arnaiz y Guerra, 2014), lo que se convierte en una alternativa de apoyo, en cualquiera de los niveles, al aprendizaje (Castevich, Dzib, Hogan, Sanders, Slovec y Yelizarov, 2015).

Considerando todo lo mencionado, se presenta el estudio llevado a cabo con una muestra de alumnos escolarizados en Institutos de Enseñanza Secundaria en la ciudad de Ceuta, donde conviven principalmente personas de cultura cristiana y musulmana. Como característica específica, la población árabe musulmana, presenta una procedencia marroquí con un altísimo nivel de 
analfabetismo y una elevada natalidad, así como un estatus socioeconómico y cultural bajo, serios problemas de enculturación y bilingüismo (integración por la comunicación) y con una presencia muy marcada de su religión, el Islam, en sus vidas (Herrera, 2000). Esto nos lleva a establecer una clara relación entre la cultura y la religión.

Los objetivos del trabajo han sido analizar los tipos y niveles en Inteligencia Emocional y Rendimiento Académico, en función de la edad, género, cultura y estrato socioeconómico. También se pretende contemplar las relaciones que se pudieran dar entre las mencionadas variables, para una población adolescente multicultural.

\section{MÉTODO}

Para llevar a cabo esta investigación se seleccionó una muestra integrada por 811 participantes que reflejan las características del contexto pluricultural de Ceuta. Se reparten entre seis centros en los que se imparte formación perteneciente a la Etapa Secundaria. El 19.6\% de la muestra cursaba el primer curso de la Enseñanza Secundaria Obligatoria (1ํㅡㄴ $\mathrm{ESO}$ ), el $20.7 \%$ cursaba $2^{\circ}$ de ESO, el $17.9 \% 3^{\circ}$ de ESO y el $12.6 \% 4^{\circ}$ de ESO. Todos ellos quedan dentro de la Educación de carácter obligatorio. A estas personas hay que sumarles el $15.4 \%$ correspondiente al primer curso de bachillerato (1은 de bachillerato) y el $6.9 \%$ al segundo curso de bachillerato. EL $6.9 \%$ restante cursaban diferentes módulos y niveles de Formación Profesional (FP). Por edad, la muestra se distribuye entre el $17.4 \%$ de entre 12 y 13 años, el $19.4 \%$ de entre 13 y 14 , el $15.8 \%$ de entre 14 y 15 , el $9.3 \%$ de entre 15 y 16 , el $15.8 \%$ entre 16 y 17 , el $7.1 \%$ de alumnos entre 17 y 18 . Estos alumnos correspondían a la muestra menor de edad, mientras que los restantes $12.1 \%$ tenían entre 18 y 25 años, $2.1 \%$ entre 26 y 40 años, mientras que sólo el 1\% tenía más de 40 años.

Considerando la cultura (que se corresponde con la religión), el grupo mayoritario es el de participantes pertenecientes a la cultura-religión musulmana. Estos constituyen el $71.6 \%$ de la muestra, mientras que el $28.4 \%$ de la misma pertenecía a la cultura-religión cristiana. Esto representa las dos culturas mayoritarias en nuestra ciudad y conforman el contexto pluricultural que la caracteriza. Describiendo la muestra, en función de la variable género, existe bastante igualdad, aunque con una ligera mayoría femenina, siendo el $46.1 \%$ varones y el resto de la muestra $(53.9 \%)$ mujeres. Con respecto a su distribución por estatus, sólo el 3.2\% de la muestra identificó su nivel como bajo. Por su parte, también fueron muy pocos los que se identificaron como pertenecientes a un nivel alto (7.2\%). El $22.3 \%$ corresponde al estatus mediobajo y el $67.2 \%$ al medio. Los participantes se seleccionaron por el método de muestreo incidental o casual, donde los integrantes de la muestra fueron aquellos alumnos que voluntariamente respondieron a los cuestionarios pasados en su momento. El error muestral fue del $3 \%$. 
Tabla 1. Muestra en función de variables sociodemográficas

\begin{tabular}{|c|c|c|c|c|}
\hline \multirow{2}{*}{$\begin{array}{l}\text { Variables } \\
\text { sociodemográficas }\end{array}$} & \multicolumn{4}{|c|}{ Media descriptiva } \\
\hline & & Media & D.T. & Rango \\
\hline Edad & $\mathrm{N}=811$ & 15.39 & 4.46 & $12-47$ \\
\hline \multirow{3}{*}{ Cultura/Religión } & & & $\mathrm{N}$ & $\%$ \\
\hline & Cristianos & & 221 & 28.4 \\
\hline & Musulmanes & & 556 & 71.6 \\
\hline \multirow[t]{2}{*}{ Género } & Varones & & 363 & 46.1 \\
\hline & Chicas & & 424 & 53.9 \\
\hline \multirow[t]{4}{*}{ Estatus } & Bajo & & 25 & 3.2 \\
\hline & Medio-Bajo & & 173 & 22.3 \\
\hline & Medio & & 521 & 67.2 \\
\hline & Alto & & 56 & 7.2 \\
\hline
\end{tabular}

Con respecto a la segunda variable, se empleó un cuestionario elaborado para evaluar la Inteligencia Emocional, como conjunto de habilidades. En este caso, el cuestionario quedó integrado por 66 ítems, divididos en 5 categorías, relacionadas con los elementos de la Inteligencia Emocional. Su fiabilidad ( $\alpha$ de Cronbach) fue de .869, mientras que la prueba de SpearmanBrown fue .767. En el apartado relacionado con la varianza factorial (AFC), los factores obtenidos fueron 5. El primero, relacionado con la Empatía, representa una varianza explicada de $15.03 \%$. El segundo está vinculado al Autoconcepto $(8.94 \%)$, el tercero al Autocontrol (5.87\%), el cuarto hace referencia a la Motivación (3.26\%), mientras que el quinto, queda vinculado al Conocimiento de sí mismos (2.85\%). Entre todos suman una varianza explicada total del $35.971 \%$. Dado que se vuelven a considerar las Habilidades Sociales, a estas cuestiones se le añaden 32 ítems para este factor ( $\alpha$ de Cronbach de .775).

Finalmente se emplearon las calificaciones, como medida del Rendimiento Académico, siguiendo el estudio de Gómez-Castro (1986).

Una vez construida la base de datos, se comenzó por la comprobación de las características de la muestra, contemplando su distribución en función de las diferentes variables sociodemográficas. Se continuó con el análisis de las propiedades psicométricas de los cuestionarios empleados. Para la presentación de los resultados, se comenzó por el análisis descriptivo, que permite la comprobación de los niveles y tipos generales para cada una de las diferentes variables. A continuación se procedió al análisis inferencial, comenzando por el análisis regresional (regresiones múltiples paso a paso 0 "stepwise"). Se consideraron las variables sociodemográficas como dependientes, junto con las variables de estudio, como variables predictoras (independientes). A continuación, se consideraron como variables criterio cada una de las variables de estudio (Inteligencia Emocional y Rendimiento Académico). Para determinar la función de regresión se consideró el último paso y, dentro de él, en el orden de entrada de las variables en la función de regresión, el valor de cada variable, su significación, el índice de regresión (R), 
el coeficiente de determinación múltiple $\left(R^{2}\right)$ y la varianza explicada de la variable más importante (la que más porcentaje - $\beta$ - explica). Para finalizar el análisis inferencial se concluyó con la prueba t de Student de diferencias de medias para muestras independientes para aquellas variables dicotómicas, mientras que para el resto se empleó el análisis de la varianza (ANOVA de un factor). Al mismo tiempo, se utilizó la prueba de Chi-cuadrado de Pearson, aprovechando los resultados obtenidos dentro de las tablas de contingencia que se hicieron para la estadística descriptiva. Para todas las pruebas realizadas se empleó un intervalo de confianza del 95\% (IC 95\%) y un nivel de significación de $p<.05$. Además, en todas las variables con más de dos alternativas de respuesta se realizaron diferentes pruebas "Pos-hoc" (DMS, Bonferroni, Turkey y Waller-Duncan). El análisis completo que ha sido posible gracias al programa informático Statistical Package for Social Sciences (SPSS 20, 2012).

\section{RESULTADOS}

Con respecto al nivel mostrado por la muestra total de alumnos de Secundaria, con respecto a la variable Inteligencia Emocional, resulta destacable que ninguno de los encuestados $(0 \%)$ reflejó niveles muy bajos en esta variable. De manera evidente, la opción mayoritaria es la que refleja bastante nivel de IE, alcanzada por el $71.2 \%$ de la muestra. Posteriormente, el resto de encuestados se distribuye de forma muy parecida entre la opción de mayor nivel de IE (con 13.42\%) y la que refleja poca IE (15.5\%). Esta situación se puede observar a través del gráfico que aparece a continuación:

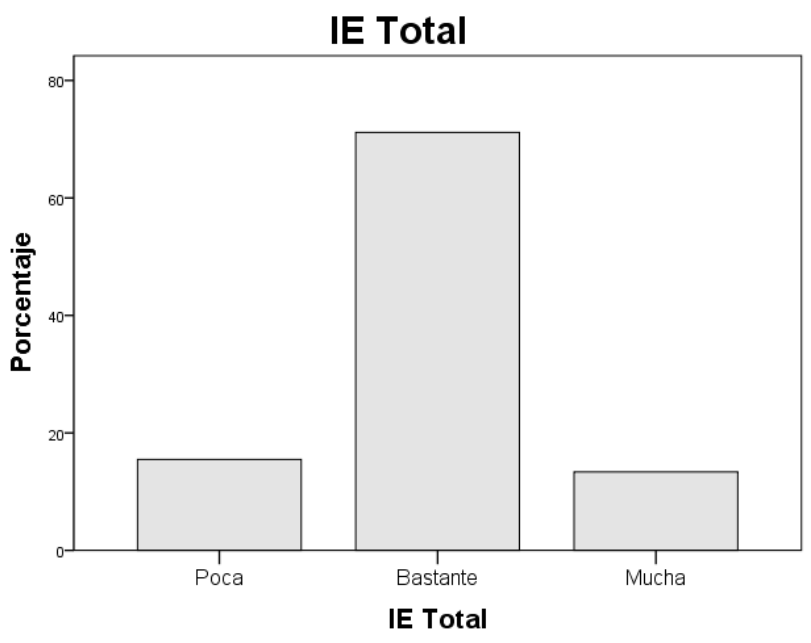

Gráfico 1. IE Total

Con respecto a la Inteligencia Emocional, en sus puntuaciones totales, el grupo de 16-17 años presenta los niveles más elevados (su media es 129.64). El siguiente, por nivel de IE, sería el grupo entre 17-18 años (125.53), seguido, a poca distancia, por el grupo de entre 18-25 (su media es de 120.62) y este, a su vez, por el de entre 12-13 años (117.08). A continuación, se produce un descenso, alcanzando el grupo de entre 15-16 años una media de 113.15. Lo 
mismo ocurre con el grupo de entre 25-39 años (112.17) y los de entre 13-14 años (112.05). Los resultados más bajos aparecen en los grupos de entre 1415 años (110.87) y de 40 o más (110.43), en el que se alcanzan los resultados más bajos. En el caso de la Empatía (52.01), el Autoconcepto (21.51), el Autocontrol (28.92), la Motivación (19.34) y el Conocimiento de sí mismos (8.47) el grupo de 16-17 años es el que alcanza resultados más altos. En el caso de las HHSS Totales, el grupo de entre 18-25 años (60.42) es el que obtiene resultados más altos. Ligeramente menos (56.63) aparecen el alumnado entre $12-13$ años y el de entre 16 y 17 (55.18). Le siguen los grupos de 40 o más (54.83), 15-16 (54.44) y 17-18 (54.08). El siguiente por nivel en este factor es el grupo de 13 a 14 años (53.85), seguido por el de 14-15 (53.76). El nivel más bajo (52.06) lo alcanza el alumnado de entre 25-39. Todo esto cobra sentido considerando el Análisis de la Varianza (ANOVA de un factor). En IE Total $(p=.000)$ y en todos sus factores (Empatía $-p=.000$-, Autoconcepto $-p=.000-$, Autocontrol $-p=.000-$, Motivación $-p=.000$ y Conocimiento de sí mismos $p=.000-)$ también aparecen diferencias estadísticamente significativas, algo que también ocurre en el factor HHSS $(p=.009)$ y en la subcategoría Enfado $(p=.002)$. Por contra se descartó la significación estadística en las subcategorías Opiniones $(p=.077)$, Asertividad $(p=.090)$, Peticiones $(p=.070)$ y Disconformidad $(p=.118)$, pertenecientes a las HHSS.

En el caso de la Inteligencia Emocional, ocurre lo mismo que lo comentado en el caso de la ansiedad y sus factores: aparecen diferencias favorables a las chicas, que reflejan niveles superiores (su media es de 120.66) que los varones (113.88). Lo mismo ocurre en diferentes factores de esta variable (Empatía los hombres 45.04 y las mujeres 49.14 , Autocontrol 24.60 en varones y 25.76 en mujeres y Conocimiento de sí mismos las mujeres 7.84 y los varones 7.21). No obstante, con respecto a las Habilidades Sociales, se puede ver una gran igualdad. A pesar de ello, los varones tienen un nivel ligeramente superior a las chicas, tal y como reflejan las medias de ambos (56.99 es la media de los varones y 54.05 la de las chicas). La prueba t de Student de diferencias de medias para muestras independientes, demuestra diferencias estadísticamente significativas en IE Total $(p=.000)$, en Empatía $(p=.000)$, Autocontrol $(p=.037)$ y Conocimiento de sí mismos $(p=.000)$, siendo las chicas las que evidencian puntuaciones superiores. Se descartó la significación estadística en Autoconcepto $(p=.068)$, Motivación $(p=.351)$, HHSS $(p=.968)$, Opiniones $(p=.379)$, Disconformidad $(p=.252)$, Asertividad $(p=.643)$, Enfado $(p=.958)$ y Peticiones $(p=.573)$.

En función de la cultura/religión, los resultados reflejan que el alumnado perteneciente a la cultura/religión musulmana manifiesta niveles más bajos en la IE (la media de los cristianos es 123.85 mientras que los musulmanes alcanzan 115.07). Esto se ve reforzado por las puntuaciones de Empatía (49.54 cristianos y musulmanes 46.35 , Autoconcepto (cristianos 20.68 y musulmanes 19.97), Autocontrol (cristianos 26.84 y musulmanes 24.59), Motivación (cristianos 18.87 y musulmanes 16.73) y Conocimiento de sí mismos (los musulmanes 7.43 y los cristianos 7.89). En el caso de las Habilidades Sociales Totales, sólo se puede destacar una enorme igualdad, ya que cristianos y musulmanes tienen una media cercana. Así los cristianos alcanzan un 56.11 de media, mientras que en los musulmanes es de 54.79. Sólo en el caso de la subcategoría Peticiones aparecen diferencias algo más evidentes (los 
cristianos presentan un nivel superior -8.90- a los musulmanes -8.16-). Considerando la prueba t de Student, con la variable Cultura/Religión como factor y el resto de las variables mencionadas como dependientes, se pueden afirmar las diferencias estadísticamente significativas en IE Total $(p=.000)$, así como en todos sus factores. Es lo que ocurre con la Empatía $(p=.000)$, el Autoconcepto $(p=.027)$, el Autocontrol $(p=.000)$, la Motivación $(p=.000)$ y el Conocimiento de sí mismos $(p=.013)$. En todos los casos, los cristianos evidencian niveles más elevados en este conjunto de habilidades emocionales. Sin embargo, dentro de las HHSS, sólo se encontraron diferencias significativas en el subfactor Peticiones $(p=.008)$, en el que los cristianos reflejan puntuaciones más altas. No fueron estadísticamente significativas en el factor HHSS $(p=.230)$ y el resto de sus categorías: manifestación de Opiniones $(p=.717)$, la Disconformidad $(p=.479)$, la Asertividad $(p=.116)$ y Enfado $(p=.121)$.

Para el estatus, se observa un aumento de IE en los niveles intermedios. De esta manera, el estatus medio ocupa la primera posición (con una media de 120.84). Le sigue el estatus medio-bajo (media de 113.64). A continuación vendría el alto (su media es de 109.16) y el bajo (100.42), que ocupa el último puesto. El Análisis de la Varianza (ANOVA de un factor), considerando la variable Estatus socio-económico y cultural demuestra diferencias estadísticamente significativas en IE Total $(p=.000)$ y casi todos sus factores (Empatía - $p=.000-$, Autoconcepto $-p=.000-$, Autocontrol $-p=.000-$ y Conocimiento de sí mismos $-p=.001-)$. Todo lo contrario ocurre con los factores Motivación ( $p=.199)$, HHSS $(p=.218)$ y los subfactores Asertividad $(p=.284)$, Enfado $(p=.107)$, Peticiones $(p=.070)$, Opiniones $(p=.047)$ y Disconformidad $(p=.058)$.

Tabla 2. ANOVA IE por variables sociodemográficas

\begin{tabular}{|c|c|c|c|c|c|c|c|}
\hline Variable & $\mathbf{N}$ & \multicolumn{2}{|c|}{ Perdidos } & Media & Des. Tip. & $\bar{F}$ & $\mathbf{P}$ \\
\hline \multicolumn{8}{|c|}{ Edad } \\
\hline & 103 & 35 & $12-13$ & 117.08 & 20.787 & & \\
\hline & 150 & 4 & $13-14$ & 112.05 & 20.975 & & \\
\hline & 124 & 1 & $14-15$ & 110.87 & 21.010 & & \\
\hline Inteligencia & 72 & 2 & $15-16$ & 113.15 & 23.385 & & \\
\hline Emocional & 119 & 6 & $16-17$ & 129.64 & 15.501 & 10.531 & .000 \\
\hline \multirow[t]{4}{*}{ Total } & 53 & 3 & 17-18 & 125.53 & 15.956 & & \\
\hline & 93 & 3 & $18-25$ & 120.62 & 21.230 & & \\
\hline & 17 & 0 & $25-39$ & 112.17 & 21.777 & & \\
\hline & 8 & 0 & 40 o más & 110.43 & 14.617 & & \\
\hline \multirow{9}{*}{$\begin{array}{l}\text { Habilidades } \\
\text { Sociales Total }\end{array}$} & 91 & 47 & $12-13$ & 56.63 & 11.856 & \multirow{9}{*}{2.557} & \multirow{9}{*}{.009} \\
\hline & 132 & 22 & $13-14$ & 53.85 & 12.668 & & \\
\hline & 111 & 14 & $14-15$ & 53.76 & 11.771 & & \\
\hline & 70 & 4 & $15-16$ & 54.44 & 12.725 & & \\
\hline & 107 & 18 & $16-17$ & 55.18 & 14.719 & & \\
\hline & 52 & 4 & $17-18$ & 54.08 & 12.292 & & \\
\hline & 88 & 8 & $18-25$ & 60.42 & 12.096 & & \\
\hline & 16 & 1 & $25-39$ & 52.06 & 14.083 & & \\
\hline & 6 & 2 & 40 o más & 54.83 & 13.862 & & \\
\hline
\end{tabular}




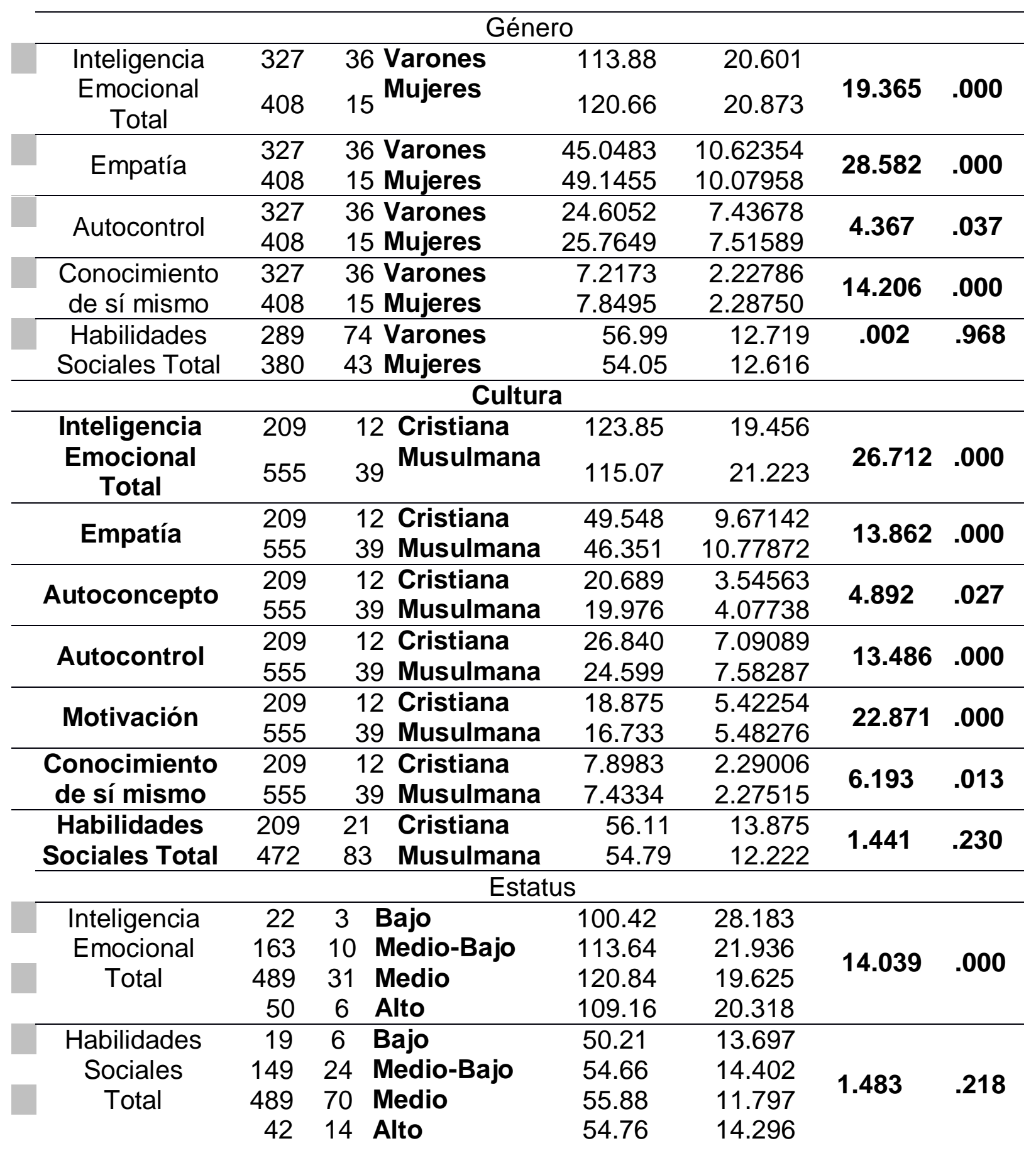

La variable Inteligencia Emocional Total se encuentra también bajo la influencia de varios factores. De todos ellos, el que tiene un coeficiente correlación estandarizado superior es la media del Rendimiento Académico que representa el $40.2 \%(\beta=.402)$, actuando de manera directamente proporcional. De esta forma, se entiende un incremento de las puntuaciones en esta variable entre los sujetos con un Rendimiento Académico más elevado. También actúan de manera positiva la Edad siendo superior las puntuaciones cuanto más alta es esta, el género siendo la puntuaciones superiores entre las mujeres. La Cultura/Religión también actúa de manera negativa siendo más altas las 
puntuaciones otros los cristianos. Entre todas las variables predictoras alcanzan un coeficiente de determinación del $43.4 \%\left(R^{2}=.434\right)$. La función de regresión y los coeficientes aparecen a continuación:

Inteligencia Emocional Total $=37.523+5.658$ (Media Rendimiento Total) + 2.575 (Edad) + 5.562 (Género) - 5.471 (Cultura) - 0,215 (Miedo Desconocido) $+2,807$ (Sexo)

Tabla 3. Coeficientes de la regresión para la Inteligencia Emocional Total

\begin{tabular}{lcccr}
\hline \multicolumn{1}{c}{$\mathbf{R}=\mathbf{. 6 5 9}$} & $\mathbf{R}^{2}=\mathbf{4 3 4}$ & $\mathbf{F}=\mathbf{4 1 . 6 9 0}$ & \multicolumn{2}{c}{$\mathbf{p = . 0 0 0}$} \\
\hline \multicolumn{1}{c}{ Variables } & $\mathbf{B}$ & $\boldsymbol{\beta}$ & $\mathbf{t}$ & $\mathbf{P}$ \\
Constante General & 37.523 & & 5.625 & .001 \\
Iedia Rendimiento & 5.658 & .402 & 12.076 & .001 \\
dad & 2.575 & .222 & 6.693 & .001 \\
énero & 5.562 & .134 & 4.092 & .001 \\
ultura/Religión & -5.471 & -.120 & -3.674 & .001 \\
\hline
\end{tabular}

Haciendo referencia al Rendimiento, establecido por las calificaciones recibidas por el alumnado (nota media), el resultado más habitual (33.7\%) es el notable (entre 7 y menos de 8.5). La siguiente calificación, por porcentaje, es el "bien" $(27.5 \%)$. A continuación aparecen las calificaciones de aprobados (con un $17.2 \%$ ) y sobresaliente (11.1\%). La calificación con porcentajes más bajos (6.2\%) es el suspenso (entre 1 y 4.5). Todo ello se resume en el gráfico que se presenta:

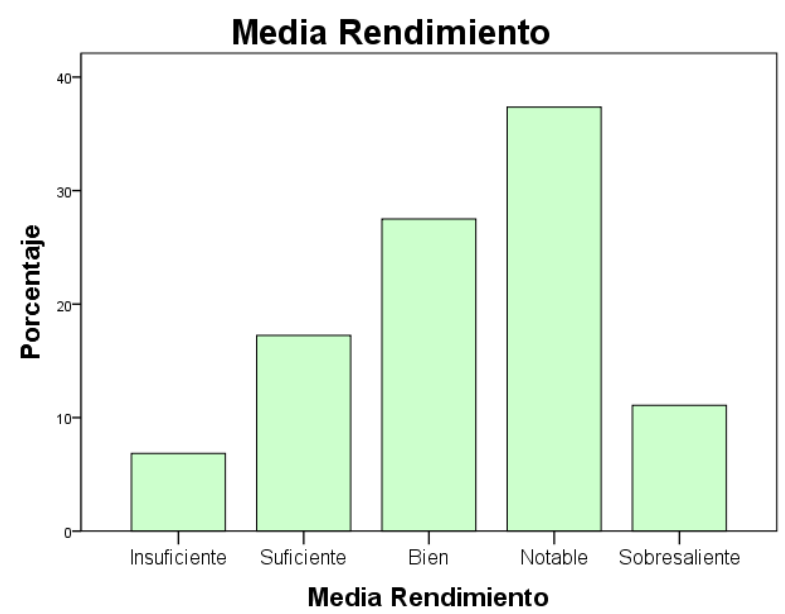

Gráfico 2. Media Rendimiento Secundaria 
Considerando la variable Rendimiento, se inicia la descripción del comportamiento de la muestra de alumnos de Secundaria en función de la variable sociodemográfica Edad. El grupo con la media más alta es el de entre 16-17 años (7.34) que presenta mayor rendimiento que el grupo de 12-13 años (6.89), al que sigue el de entre 13-14 (6.65). A este le sigue el de 18-25 años (6.48) y muy próximo, (6.45) el de 17 a 18. El grupo de entre 15-16 (con una media de 6.2) supera al siguiente en su media (6.15). Los resultados medios más bajos se dan en el alumnado de 14-15 (6.11) años y, sobre todo en el de 40 o más (5.53). Una situación similar se describe en el Rendimiento en las diferentes materias. En todas ellas, el alumnado de entre 16-17 años presenta las puntuaciones más altas (Lengua Castellana 7.08, ciencias Sociales 7.36, Inglés 7.21, ciencias Naturales 7.53 y Religión/Ciudadanía 8.47). La única excepción es la materia de Matemáticas, donde el grupo de menor edad (12-13) es el que alcanza calificaciones más altas (6.58 de media). El Análisis de la Varianza (ANOVA de un factor), con la variables Edad como variable independiente y el resto de las variables como dependientes muestra diferencias estadísticamente significativas en la Media general $(p=.000)$. Lo mismo ocurre con las materias específicas: Lengua Castellana $(p=.000)$, Matemáticas $(p=.000)$, Sociales $(p=.019)$, Inglés $(p=.000)$, Naturales $(p=.000)$ y Religión $(p=.000)$.

Si se tiene en cuenta la Media del Rendimiento, en función del género, se puede comprobar una enorme igualdad entre hombres y mujeres en cuanto a las calificaciones. Así las chicas alcanzan una media de 6.82, mientras que los chicos llegan hasta 6.43. De esta manera, se puede hablar de resultados algo superiores en las chicas. No obstante, considerando la prueba t de Student de diferencias de medias para muestras independientes, se descartó la significación la Media general $(p=.667)$, Lengua Castellana $(p=.952)$, Matemáticas $(p=.257)$, Sociales $(p=.661)$, Inglés $(p=.870)$, Naturales $(p=.737)$ y Religión ( $p=.567)$.

Sin embargo, si se tiene en cuenta la Media de Rendimiento, en función de la cultura/religión, se puede decir que las calificaciones son claramente superiores entre los cristianos. La media de los cristianos es de 7.15, mientras que la de los musulmanes es de 6.45. Así, los porcentajes muestran un predominio entre los cristianos en lo que a sobresalientes (16.3\% frente al 9.3\%) y notables $(48.5 \%$ en cristianos y $33.5 \%$ en musulmanes) se refiere. Por su lado, la población cristiana tiene porcentajes menores en cuanto a suspensos (3\% frente a $7.9 \%$ ). Por lo tanto, se puede ver que los pertenecientes al grupo "cristianos" tienen un Rendimiento Académico superior, realidad que se repite en cada una de las materias (en Lengua Castellana 6.92 los cristianos y 6.17 los musulmanes, en Matemáticas, 6.48 frente a 5.93, en ciencias Sociales, los musulmanes 6.7, mientras que los cristianos 7.33, en Inglés, los cristianos 6.96 y los musulmanes 6.20, en ciencias 7.09 los cristianos y 6.32 los musulmanes y en Religión/Ciudadanía la población de cultura cristiana 8.09 y los musulmanes 7.41). Esta realidad queda confirmada por la prueba t de Student, dado que las diferencias fueron estadísticamente significativas en la Media general $(p=.000)$, en Lengua Castellana $(p=.000)$, Matemáticas $(p=.001)$, Sociales $(p=.033)$, Inglés $(p=.000)$, Naturales $(p=.000)$ y Religión $(p=.000)$. En todos los casos, son los musulmanes los que obtienen resultados más bajos.

Considerando la Media del Rendimiento, dentro de la última de las variables, se observa que las calificaciones aumentan progresivamente a 
medida que se asciende en el estatus. De esta manera, en el estatus alto es en el que aparecen niveles superiores con el segundo mayor porcentaje de sobresalientes (11.5\%) y notables (51.9\%), seguido del medio con menor porcentaje de notables $(41.7 \%)$ pero más sobresalientes (12.5\%). Estos presentan una media de 7.08 y 6.8 respectivamente. Las calificaciones descienden bastante para los otros dos grupos. El medio bajo (6.11) y el bajo (5.33) presentan los porcentajes más altos de suspensos (14.1\% y $27.3 \%$ respectivamente). El estatus alto es en el que aparecen los mejores resultados también en Lengua Castellana (6.84), en Matemáticas (6.46), en ciencias Sociales (7.44), en lengua extranjera (Inglés donde la media es 6.75), en ciencias Naturales (7.03) y en Religión/Ciudadanía (8.98). Una vez hecha esta primera aproximación, el Análisis de la Varianza (ANOVA de un factor), demostró diferencias estadísticamente significativas en la Media general $(p=.000)$, así como en las áreas específicas: Lengua Castellana $(p=.000)$, Matemáticas $(p=.000)$, Sociales $(p=.000)$, Inglés $(p=.000)$, Naturales $(p=.000)$ y Religión $(p=.000)$.

Tabla 4. ANOVA Rendimiento por variables sociodemográficas

\begin{tabular}{|c|c|c|c|c|c|c|c|}
\hline Variable & $\mathbf{N}$ & Perc & didos & Media & Des. Tip. & $\mathbf{F}$ & $\mathbf{P}$ \\
\hline \multicolumn{8}{|c|}{ Edad } \\
\hline & 127 & 10 & 12-13 & 6.89948 & 1.644860 & \multirow{9}{*}{7.384} & \multirow{9}{*}{.000} \\
\hline & 153 & 1 & $13-14$ & 6.65741 & 1.625072 & & \\
\hline & 120 & 5 & $14-15$ & 6.11806 & 1.417082 & & \\
\hline Media & 72 & 2 & $15-16$ & 6.20023 & 1.473349 & & \\
\hline Rendimiento & 122 & 3 & $16-17$ & 7.34563 & 1.193941 & & \\
\hline \multirow{4}{*}{ Académico } & 43 & 13 & $17-18$ & 6.45736 & .977473 & & \\
\hline & 72 & 24 & $18-25$ & 6.48762 & 1.616180 & & \\
\hline & 12 & 5 & $25-39$ & 6.15972 & 1.057953 & & \\
\hline & 5 & 3 & 40 o más & 5.53333 & 1.340605 & & \\
\hline \multicolumn{8}{|c|}{ Género } \\
\hline Media & 338 & 25 & Varones & 6.43013 & 1.539395 & \multirow[t]{2}{*}{.185} & \multirow[t]{2}{*}{.667} \\
\hline Rendimiento & 387 & 36 & Mujeres & 6.82845 & 1.476712 & & \\
\hline \multicolumn{8}{|c|}{ Cultura } \\
\hline Media & 202 & 19 & Cristiana & 7.15000 & 1.424128 & \multirow{2}{*}{31.291} & \multirow{2}{*}{.000} \\
\hline Rendimiento & 516 & 39 & Musulmana & 6.45945 & 1.511363 & & \\
\hline \multirow{2}{*}{$\begin{array}{c}\text { Lengua } \\
\text { castellana }\end{array}$} & 202 & 19 & Cristiana & 6.92327 & 1.767676 & \multirow{2}{*}{24.988} & \multirow{2}{*}{.000} \\
\hline & 516 & 39 & Musulmana & 6.17742 & 1.809304 & & \\
\hline \multirow{2}{*}{ Matemáticas } & 202 & 19 & Cristiana & 6.48515 & 1.939964 & \multirow{2}{*}{11.074} & \multirow{2}{*}{.001} \\
\hline & 516 & 39 & Musulmana & 5.93488 & 2.012337 & & \\
\hline \multirow{2}{*}{ Sociales } & 202 & 19 & Cristiana & 7.33663 & 1.762812 & \multirow{2}{*}{15.756} & \multirow{2}{*}{.000} \\
\hline & 516 & 39 & Musulmana & 6.70339 & 1.980883 & & \\
\hline \multirow{2}{*}{ Inglés } & 202 & 19 & Cristiana & 6.96535 & 1.883098 & \multirow{2}{*}{19.534} & \multirow{2}{*}{.000} \\
\hline & 516 & 39 & Musulmana & 6.20494 & 2.142447 & & \\
\hline \multirow{2}{*}{ Naturales } & 202 & 19 & Cristiana & 7.09802 & 1.906077 & \multirow{2}{*}{24.064} & \multirow{2}{*}{.000} \\
\hline & 516 & 39 & Musulmana & 6.32016 & 1.912273 & & \\
\hline \multirow{2}{*}{ Religión } & 202 & 19 & Cristiana & 8.09158 & 1.670189 & \multirow{2}{*}{19.969} & \multirow{2}{*}{.000} \\
\hline & 516 & 39 & Musulmana & 7.41589 & 1.877689 & & \\
\hline
\end{tabular}




\begin{tabular}{|c|c|c|c|c|c|c|c|}
\hline \multicolumn{8}{|c|}{ Estatus } \\
\hline \multirow{4}{*}{$\begin{array}{c}\text { Media } \\
\text { Rendimiento }\end{array}$} & 22 & 3 & Bajo & 5.38864 & 2.015373 & \multirow{4}{*}{17.091} & \multirow{4}{*}{.000} \\
\hline & 156 & 17 & Medio-Bajo & 6.11047 & 1.594503 & & \\
\hline & 489 & 32 & Medio & 6.84707 & 1.392799 & & \\
\hline & 52 & 4 & Alto & 7.08654 & 1.381283 & & \\
\hline \multirow{4}{*}{$\begin{array}{l}\text { Rendimiento } \\
\text { Lengua } \\
\text { castellana }\end{array}$} & 22 & 3 & Bajo & 5.50000 & 2.304240 & \multirow{4}{*}{10.171} & \multirow{4}{*}{.000} \\
\hline & 156 & 17 & Medio-Bajo & 5.81090 & 1.882083 & & \\
\hline & 489 & 32 & Medio & 6.57587 & 1.750641 & & \\
\hline & 52 & 4 & Alto & 6.84615 & 1.576601 & & \\
\hline \multirow{4}{*}{ Matemáticas } & 22 & 3 & Bajo & 4.95455 & 2.011332 & \multirow{4}{*}{9.121} & \multirow{4}{*}{.000} \\
\hline & 156 & 17 & Medio-Bajo & 5.52244 & 1.970216 & & \\
\hline & 489 & 32 & Medio & 6.28609 & 1.943571 & & \\
\hline & 52 & 4 & Alto & 6.46154 & 1.994714 & & \\
\hline \multirow{4}{*}{ Sociales } & 22 & 3 & Bajo & 5.77273 & 2.524769 & \multirow{4}{*}{12.077} & \multirow{4}{*}{.000} \\
\hline & 156 & 17 & Medio-Bajo & 6.25321 & 2.067020 & & \\
\hline & 489 & 32 & Medio & 7.09857 & 1.814609 & & \\
\hline & 52 & 4 & Alto & 7.44231 & 1.526414 & & \\
\hline \multirow{4}{*}{ Inglés } & 22 & 3 & Bajo & 4.86364 & 2.396336 & \multirow{4}{*}{7.435} & \multirow{4}{*}{.000} \\
\hline & 156 & 17 & Medio-Bajo & 6.02564 & 2.161835 & & \\
\hline & 489 & 32 & Medio & 6.57975 & 2.032971 & & \\
\hline & 52 & 4 & Alto & 6.75000 & 2.008560 & & \\
\hline \multirow{4}{*}{ Naturales } & 22 & 3 & Bajo & 5.38636 & 2.023268 & \multirow{4}{*}{10.027} & \multirow{4}{*}{.000} \\
\hline & 156 & 17 & Medio-Bajo & 5.98077 & 2.037238 & & \\
\hline & 489 & 32 & Medio & 6.72802 & 1.844021 & & \\
\hline & 52 & 4 & Alto & 7.03846 & 1.899035 & & \\
\hline \multirow{4}{*}{ Religión } & 22 & 3 & Bajo & 5.85455 & 2.43559 & \multirow{4}{*}{14.72} & \multirow{4}{*}{.000} \\
\hline & 156 & 17 & Medio-Bajo & 7.06987 & 2.00860 & & \\
\hline & 489 & 32 & Medio & 7.81411 & 1.67920 & & \\
\hline & 52 & 4 & Alto & 7.98077 & 1.73194 & & \\
\hline
\end{tabular}

Los resultados que a continuación se presentan, que reflejan el paso final del método elegido para la realización de las regresiones, ponen de manifiesto que la media del Rendimiento Académico (media de calificaciones) está bajo la influencia de varias variables. De todas ellas en la que ejerce un poder más determinante es la Inteligencia Emocional Total, cuyo coeficiente de regresión estandarizado es del $35.1 \% \quad(\beta=.351)$. Esta variable actúa de manera directamente proporcional. Por otra parte, también ejercen influencia las variables Estatus, la Edad, el Conocimiento de sí mismos y la Motivación (IE) y la Cultura/Religión.

De todas ellas, solamente dos actúan en sentido inverso (inversamente proporcional), siendo la Edad y la Cultura/Religión. Esto indica que a medida que ascendemos en la Edad y entre los sujetos pertenecientes a la cultura musulmana es inferior la media del Rendimiento. El estatus y el resto variables socio demográficas actúan de manera positiva. Lo mismo ocurre con los factores de la IE. El resto de variables quedan excluidas, explicando entre todas las predictoras el $37.7 \%$ de la varianza total $\left(R^{2}=.377\right)$. La influencia de 
estas variables es intensa, tal y como indica el coeficiente de correlación $(\mathrm{R}=.614)$. La función descrita sería la siguiente, la cual se acompaña con una tabla con los coeficientes más relevantes:

Media Rendimiento $=1.588+.025$ (Inteligencia Emocional Total) +.348 (Estatus) + .078 (Conocimiento de sí mismos) + .029 (Motivación) - .276 (Cultura/Religión) - .042 (Edad)

Tabla 5. Coeficientes de la regresión para la Media de Rendimiento

\begin{tabular}{llccc}
\hline \multicolumn{1}{c}{$\mathbf{R}=\mathbf{c} \mathbf{6 1 4}$} & $\mathbf{R}^{2}=. \mathbf{3 7 7}$ & & $\mathbf{F}=\mathbf{3 2 . 8 1 8}$ & \multicolumn{2}{c}{$\mathbf{p = . 0 0 0}$} \\
\hline \multicolumn{1}{c}{ Variables } & $\mathbf{B}$ & $\boldsymbol{\beta}$ & $\mathbf{t}$ & \multicolumn{1}{c}{$\mathbf{P}$} \\
$\quad$ Constante General & 1.588 & & 2.994 & .003 \\
IE Total & .025 & .351 & 7.548 & .000 \\
Estatus & .348 & .142 & 4.204 & .000 \\
Conocimiento sí mismo & .078 & .123 & 3.327 & .001 \\
Motivación & .029 & .109 & 2.777 & .006 \\
Cultura/Religión & -.276 & -.085 & -2.516 & .012 \\
Edad & -.042 & -.082 & -1.998 & .046 \\
\hline
\end{tabular}

El Análisis de la Varianza (ANOVA de un factor), considerando la variable Inteligencia Emocional (IE Total) como factor y el resto de las variables mencionadas como dependientes. Las diferencias fueron estadísticamente significativas en la Media del Rendimiento Académico $(p=.000)$, así como en Lengua Castellana $(p=.000)$, Matemáticas $(p=.000)$, Sociales $(p=.033)$, Inglés $(p=.000)$, Naturales $(p=.000)$ y Religión $(p=.000)$. En todos los casos se describe una relación directamente proporcional entre la IE y el Rendimiento Académico, del mismo modo que ocurre con cada una de las materias. El análisis post hoc permitió establecer diferencias significativas entre todos los grupos.

Tabla 6. ANOVA Rendimiento por IE Total Secundaria

\begin{tabular}{|c|c|c|c|c|c|c|c|}
\hline Variable & $\mathbf{N}$ & Perc & idos & Media & Des. Tip. & $\mathbf{F}$ & $\mathbf{P}$ \\
\hline \multicolumn{8}{|c|}{ IE Total } \\
\hline \multirow{3}{*}{$\begin{array}{c}\text { Media } \\
\text { Rendimiento }\end{array}$} & 102 & 15 & Poca IE & 5.33946 & 1.406308 & \multirow{3}{*}{97.113} & \multirow{3}{*}{.000} \\
\hline & 496 & 42 & $\begin{array}{l}\text { Bastante } \\
\text { IE }\end{array}$ & 6.70351 & 1.342374 & & \\
\hline & 91 & 10 & Mucha IE & 8.02729 & 1.247408 & & \\
\hline \multirow{3}{*}{$\begin{array}{c}\text { Rendimiento } \\
\text { Lengua } \\
\text { castellana }\end{array}$} & 102 & 15 & Poca IE & 5.08824 & 1.664141 & \multirow{3}{*}{57.304} & \multirow{3}{*}{.000} \\
\hline & 496 & 42 & $\begin{array}{l}\text { Bastante } \\
\text { IE }\end{array}$ & 6.44950 & 1.717211 & & \\
\hline & 91 & 10 & Mucha IE & 7.69341 & 1.595327 & & \\
\hline Matemáticas & $\begin{array}{l}102 \\
496\end{array}$ & $\begin{array}{l}15 \\
42\end{array}$ & $\begin{array}{l}\text { Poca IE } \\
\text { Bastante }\end{array}$ & $\begin{array}{l}4.90882 \\
6.05988\end{array}$ & $\begin{array}{l}1.818067 \\
1.929365\end{array}$ & 40.711 & .000 \\
\hline
\end{tabular}




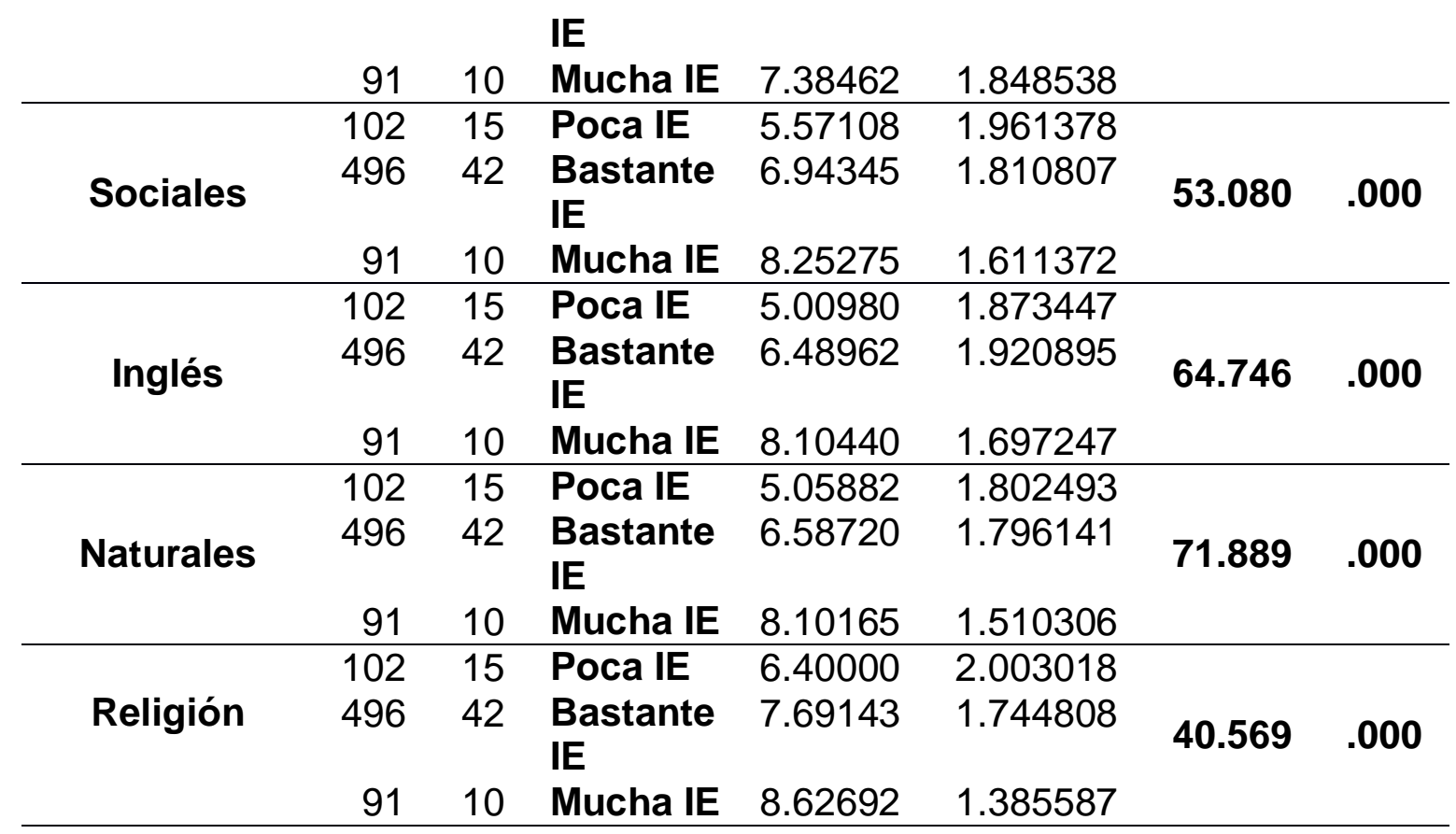

\section{DISCUSIÓN}

Se inicia este apartado con la influencia de la edad en el Rendimiento Académico. De manera contraria a lo que se esperaba, se encontraron diferencias significativas en cuanto al Rendimiento en todas las materias, así como también en la Media del Rendimiento académico. En esta ocasión, se vuelve a incidir en la relación entre el curso y la edad, para justificar estas diferencias. Se vuelve a mencionar el trabajo de Pulido y Herrera (2015) como ejemplo en el que se pueden ver diferencias considerando esta variable. Si se considera el género, no se encontraron diferencias significativas entre ambos, ni desde el punto de vista de la Media, ni tampoco en función de cada una de las materias. En este sentido, las diferencias entre géneros en cuanto al Rendimiento se pueden observar en Pulido y Herrera (2015).

Con respecto al Rendimiento en función de la cultura/religión del individuo, tal y como se pensaba, se comprueban diferencias estadísticamente significativas en cuanto al Rendimiento (Media), así como en todas y cada una de las materias, por lo que se podría decir que es un factor influyente en esta variable. En todos los casos, el grupo de alumnos pertenecientes a la cultura/religión cristiana-occidental obtienen mejores resultados. Esta realidad ya fue descrita en trabajos anteriores (Pulido y Herrera, 2015). Con respecto al Rendimiento en función del estatus, también se encontraron diferencias significativas en el Rendimiento. Esto se cumple para la Media de Rendimiento y para cada una de las materias. En todos los casos, las calificaciones aumentan a medida que lo hace el estatus socio-económico y cultural. Esta realidad se encontró también en experiencias previas (Pulido y Herrera, 2015).

Se continúa con la siguiente variable, la IE en función de la edad, que vuelve a ser un factor determinante, dado que se pueden recoger diferencias estadísticamente significativas, tanto en el nivel general de IE como en los 
factores que la conforman (tipos), incluidas las HHSS. Se prosigue con la Inteligencia Emocional en función del género, donde se volvieron a encontrar diferencias estadísticamente significativas entre ambos. En IE Total, en Empatía, Autocontrol y Conocimiento de sí mismos, las chicas evidencian puntuaciones superiores (Downey, Johnston, Hansen, Birney y Stough, 2013; Billings, Downey, Lomas, Lloyd \& Stough, 2014; Cabello \& Fernandez-Berrocal, 2015; Pulido y Herrera, 2015 y Caballero-Dominguez et al., 2015). Se prosigue en función de la cultura/religión. Se puede considerar que la cultura/religión es una variable determinante en cuanto a las puntuaciones en los totales y también en sus factores, excepto en las Habilidades Sociales. En cada uno de los casos en los que se recogieron diferencias significativas fueron los cristianos los que obtuvieron resultados superiores.

En otras experiencias, la cultura también fue un elemento a considerar (Pulido y Herrera, 2015). Se prosigue esta discusión contemplando el comportamiento de la IE en función del estatus, variable determinante en cuanto a las puntuaciones en los totales y también en sus factores. En este caso, los resultados más altos se dieron en el estatus. Coincidiendo con la presencia de diferencias, en función del estatus aparecen los trabajos de Alonso y Román (2014) y Pulido y Herrera (2015). A pesar de ello, la dirección de estas diferencias no es la misma que la comentada en estos trabajos, ya que presentan mayores capacidades emocionales aquellas personas pertenecientes a estatus superiores.

Con respecto a la interacción con el Rendimiento Académico, tal y como esperábamos, encontramos diferencias estadísticamente significativas tanto para la Media del Rendimiento, como para todas y cada una de las materias escolares. En este sentido, en todos los casos los sujetos que tenían mayor nivel de Inteligencia Emocional, presentaban mejores resultados académicos. Estos resultados vienen a confirmar los encontrados por diferentes autores en los que se demuestra que las competencias emocionales son fundamentales para la mejora del rendimiento académico (Gorostiaga y Balluerka, 2014), siendo directamente proporcionales las relaciones entre las puntuaciones en inteligencia emocional y el rendimiento académico (Codier y Odell, 2014 y Hanin \& Van Nieuwenhoven, 2016).

\section{CONCLUSIONES}

En Rendimiento Académico, los resultados más altos son para el alumnado de entre 16 y 17 años, dándose diferencias con el resto de grupos de edad. La vinculación entre el curso y la edad es un claro justificante de estas diferencias, a pesar de que no siguen una progresión lógica. Este grupo de edad se corresponde con el alumnado perteneciente a los bachilleratos, modalidad no obligatoria, lo que hace que el alumnado inscrito en ésta tenga una mayor predisposición a trabajar. Considerando el género, no se encontraron diferencias significativas entre ambos, ni en el nivel (total) ni en los tipos (materias), tal y como se pensaba en un principio. La asignatura en la que se registran resultados mejores es en Religión, en ambos grupos por igual. Las diferencias en los patrones de crianza no justificarían la aparición de diferencias en cuanto al rendimiento académico. 
La cultura es un factor a tener en cuenta en el rendimiento en las asignaturas, del mismo modo que en el rendimiento general. Como justificante se puede volver a mencionar lo comentado en el trabajo, ya mencionado, de Alonso y Román (2014), así como las dificultades para la integración socio educativa (Oropesa et al., 2014 y Cervini et al., 2014) y las diferencias de estatus socioeconómico y cultural (Cervini et al., 2014). También destacan las importantes diferencias en cuanto a la lengua materna entre musulmanes y cristianos (Roa, 2006 y Pulido y Herrera, 2015). Todos estos elementos se unen para provocar importantes problemas de integración socioeducativa del alumnado que no tiene el castellano como lengua madre, lengua vehicular en el ámbito educativo (Pulido y Herrera, 2015). De todos estos elementos, se hace especial énfasis en las diferencias en el lenguaje que adquieren ambos grupos culturales. La población cristiana desarrolla el castellano como lengua madre, sin embargo, en la población musulmana es el dariya, un dialecto del árabe el que desempeña esta función de primera lengua. Dado que el castellano es la lengua vehicular dentro del ámbito educativo, existe una mayor predisposición a la comprensión por parte del alumnado cristiano. Esta realidad facilita de manera evidente el desarrollo curricular, haciéndolo más complejo para los musulmanes, dado que suelen aparecer mayores dificultades para la comprensión verbal.

En IE, aparecen puntuaciones más bajas entre los más jóvenes. Esto permite que se pueda decir que se experimenta un ascenso progresivo a medida que se va aumentando la edad, hasta alcanzar el periodo adulto. Dentro del factor Habilidades Sociales, también se encontraron diferencias estadísticamente significativas (HHSS), donde también se puede entender un aumento progresivo en las puntuaciones a medida que asciende la edad. Para todo esto, se puede destacar el proceso de desarrollo socio-emocional del sujeto. Este crecimiento progresivo provoca importantes cambios en todas las capacidades que forman parte de la inteligencia emocional, incluidas las HHSS (Cazalla-Luna \& Molero, 2014; Gomes y Pereira, 2014 y Pulido y Herrera, 2015).

Considerando la Inteligencia Emocional en función del género, se volvieron a dar diferencias significativas entre ambos. Alejados de aquellas concepciones basadas en criterios de tipo biológico (Pinto, Dutra, Filgueiras, Juruena \& Stingel, 2013), se destacan las relacionadas con patrones de socialización y desempeño de distintos roles diferentes para ambos grupos (Soriano \& González, 2013 y Pulido y Herrera, 2015). Existe una tendencia a hacer que los varones repriman y no muestren sus manifestaciones emocionales, algo que no se le suele pedir a las niñas. Esta realidad, podría justificar el estereotipo de que las mujeres son más emocionales, siendo un justificante de estas diferencias en el manejo de habilidades emocionales.

Todo lo comentado cambia si se consideran las HHSS. La cultura/religión es también una variable determinante, excepto en las Habilidades Sociales. En todos los casos, fueron los musulmanes los que obtuvieron resultados inferiores, lo que demuestra que la cultura de origen influiría en las relaciones afectivas y en la constitución de competencias emocionales (Braz, Cômodo, Del Prette, Del Prette y Fontaine, 2013; Soriano \& González, 2013 y Gutiérrez \& Expósito, 2015). Al mismo tiempo, el desarrollo de estas competencias tiene una importante relación con la integración efectiva de sujetos pertenecientes a 
alguna minoría social, étnica o cultural, existiendo una relación directa entre la inteligencia emociona la cultura (Merchán, Bermejo \& González, 2014). Como explicación a estas diferencias se debe señalar que el nivel económico y sociocultural correlaciona positivamente con el estilo educativo familiar, y éste, a su vez, con el nivel de autoestima mayor que repercute en diferentes dimensiones de desarrollo: ajuste emocional, éxito social y académico (Alonso y Román, 2014). Se concluye este apartado de análisis de la influencia de las variables sociodemográficas con el comportamiento de la IE en función del estatus. Esta variable vuelve a ser determinante, tanto en las puntuaciones totales como en sus factores. Para justificar estas diferencias se ha de mencionar la clara relación entre el estatus y la cultura, algo ya descrito en Herrera (2000), lo que vuelve a hacer que se consideren el nivel económico y sociocultural, el ajuste emocional, el éxito social y las diferencias en el uso del lenguaje como claros justificantes para estas diferencias.

Se concluye con la idea de que a mayor nivel de IE mayor Rendimiento Académico, encontrando una relación positiva entre ambos. Dichas habilidades pueden actuar como factor de vulnerabilidad, influyendo negativamente en los estudiantes, no sólo dentro, sino también fuera de las situaciones escolares, influyendo sus carencias en el rendimiento académico (Gutiérrez \& Expósito, 2015), siendo la emocionalidad fundamental para llegar a un alto rendimiento, no sólo a nivel académico, sino también social, empresarial, personal... (Pacheco et al., 2015). Por esta razón, considerar y potenciar las competencias emocionales se antoja como una necesidad dentro de ámbito educativo.

\section{REFERENCIAS BIBLIOGRÁFICAS}

Alonso, J. y Román, J. M. (2014). Nivel sociocultural, prácticas educativas familiares y autoestima de los hijos en edades tempranas. Revista de Investigación Educativa, https://doi.org/10.6018/rie.32.1.173421

$32(1)$,

187-202

Aguaded, M. C. y Pantoja, M. J. (2015). Innovar desde un proyecto educativo de Inteligencia Emocional en primaria e infantil. Tendencias Pedagógicas, 26, 69-88. Recuperado de http://dialnet.unirioja.es/revista/1412/A/2015.

Azpiazu, L., Esnaola, I., \& Sarasa, M. (2015). Predictive capacity of social support on emotional intelligence in adolescence. European Journal of Education and Psychology, 8(1), 23-29. https://doi.org/10.1016/j.ejeps.2015.10.003

Billings, C., Downey, L. A., Lomas, J. E., Lloyd, J., \& Stough, C. (2014). Emotional Intelligence and scholastic achievement in pre-adolescent children. Personality and Individual Differences, 65, 14-18. https://doi.org/10.1016/j.paid.2014.01.017

Braz, A. C., Cômodo, C. N., Del Prette, Z., Del Prette, A. y Fontaine, A. (2013). Habilidades sociales e intergeneracionalidad en las relaciones familiares. Apuntes de Psicología, 31(1), 77-84. 
Caballero-Dominguez, C. C., Suárez-Colorado, Y. P. \& BrugesaCarbono, H. D. (2015). Characteristics of Emotional Intelligence in a Group of Higher Education Students with and without Suicidal Ideation. Revista CES Psicología, 8(2), 138-155.

Cabello, R. \& Fernandez-Berrocal, P. (2015). Implicit theories and ability emotional intelligence. Frontiers in Psychology, 6. https://doi.org/10.3389/fpsyg.2015.00700

Castevich, J., Dzib, A., Hogan, D. L., Sanders, L., Slovec, K. y Yelizarov, D. (2015). Desarrollo del entorno personal de aprendizaje para tutoría e investigación en niveles educativos superiores. Revista Innoeduca, 1(1), 15-26.

Cazalla-Luna, N. \& Molero, D. (2014). Inteligencia emocional percibida, ansiedad y afectos en estudiantes universitarios. Revista Española de

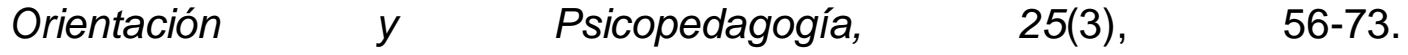
doi:10.5944/reop.vol.25.num.3.2014.13858

https://doi.org/10.5944/reop.vol.25.num.3.2014.13858

Cejudo, J. (2016). Relationship between Emotional Intelligence and mental health in School Counselors. Electronic Journal of Research in Educational Psychology, 14(1), 131-154. doi.org/10.14204/ejrep.38.15025 https://doi.org/10.14204/ejrep.38.15025

Cervini, R., Dari, N. y Quiroz, S. (2014). Estructura familiar y Rendimiento Académico en países de América Latina. Los datos del Segundo Estudio Regional Comparativo y Explicativo. Revista Mexicana de Investigación Educativa, 19(61), 569-597.

Codier, E. y Odell, E. (2014). Measured emotional intelligence ability and grade point average in nursing students. Nurse Education Today, 34, 608-612. https://doi.org/10.1016/j.nedt.2013.06.007

De Haro, J. M. y Castejón, J. L. (2014). Perceived emotional intelligence, general intelligence and earlyprofessionalsuccess: predictive and incremental validity. Anales de Psicología, 30(2), 490-498 https://doi.org/10.6018/analesps.30.2.154621

Di Giusto, C., Martín M. E., Arnaiz, A. y Guerra, P. (2014). Competencias personales y sociales en adolescentes. Revista Iberoamericana de Educación, 66, 89-104. Recuperado de http://www.rieoei.org/rie66a06.pdf.

Downey, L. A., Johnston, P. J., Hansen, K., Birney, J. y Stough, C. (2010). Investigating the mediating effects of emotional intelligence and coping on problem behaviours in adolescents. Australian Journal of Psychology, 62, 20-29. https://doi.org/10.1080/00049530903312873

Gomes, R. M. y Pereira, A., (2014). Influence of Age and Gender in Acquiring Social Skillsin Portuguese Preschool Education. Psychology, 5(2), 99-103. https://doi.org/10.4236/psych.2014.52015

Gómez-Castro, J. L. (1986). "Rendimiento escolar y valores interpersonales: Análisis de resultados en E.G.B. con el cuestionario SIV de Leonardo V. Gordon". Bordón, 262, 257-275. 
Gorostiaga, A. y Balluerka, N. (2014). Evaluación de la empatía en el ámbito educativo y su relación con la inteligencia emocional. Revista de Educación, 364, 12-38.

Gutiérrez, M. \& Expósito, J. (2015). Autoconcepto, dificultades interpersonales, habilidades sociales y conductas asertivas en adolescentes. Revista Española de Orientación y Psicopedagogía, 26(2), 42-58. doi:10.5944/reop.vol.26.num. 2.2015.15215

Hanin, V. \& Van Nieuwenhoven, C. (2016). The influence of motivational and emotional factors in mathematical learning in secondary education. Revue européenne de psychologie appliquée, 66, 127-138. doi:10.1016/j.erap.2016. 04.006

Herrera, F. (2000). La inmigración extranjera no comunitaria y la convivencia en contextos concretos: el caso de Ceuta. En Instituto de Estudios Ceutíes, Monografía de los cursos de Verano de la Universidad de Granada en Ceuta (12 ${ }^{\mathrm{a}}$ ed., pp. 357-359). Ceuta: Instituto de Estudios CeutíesUniversidad de Granada.

Merchán, I. M., Bermejo, M. L. \& González, J. D. (2014). Eficacia de un Programa de Educación Emocional en Educación Primaria. Revista de Estudios e Investigación en Psicología y Educación, 1(1), 91-99. doi:10.17979/reipe. 2014.1.1.30

Oberle, E., Schonert-Reichl, K. A. y Zumbo, B. D. (2011). Life satisfaction in early adolescence: Personal, neighborhood, school, family, and peer influences. Journal of Youth and Adolescence, 40, 889-901. https://doi.org/10.1007/s10964-010-9599-1

Oropesa, F., Moreno, C., Pérez, P. \& Muñoz-Tinoco, V. (2014). Rutinas de tiempo libre: oportunidad y riesgo en la adolescencia. Cultura y Educación, 26(1),159-183. https://doi.org/10.1080/11356405.2014.908670

Pacheco H, P., Villagrán R, S., \& Guzmán A, C. (2015). Estudio del campo emocional en el aula y simulación de su evolución durante un proceso de enseñanza-aprendizaje para cursos de ciencias. Estudios pedagógicos, 41(1), 199-217. https://doi.org/10.4067/S0718-07052015000100012

Pinto, B. M. D. C., Dutra, N. B., Filgueiras, A., Juruena, M. F. P., \& Stingel, A. M. (2013). Diferenças de gênero entre universitários no reconhecimento de expressões faciais emocionais. Avances en Psicología Latinoamericana, 31(1), 200-222.

Pulido, F. y Herrera, F. (2015). Miedo y rendimiento académico en el contexto pluricultural de Ceuta. Revista de Investigación Educativa, 34(1), 185203.doi: http://dx.doi.org/10.6018/rie.34.1.207221

Pulido, F., Herrera, F. (2016). Diferencias por género en una validación inicial de un instrumento en construcción para evaluar la inteligencia emocional en una muestra de alumnos de secundaria. Tendencias Pedagógicas, 28, 99-114.

Roa, J. M. (2006). Rendimiento escolar y "situación diglósica" en una muestra de escolares de educación primaria en Ceuta. Revista Electrónica de Investigación Educativa, 8, 1. 
Siqués, C. \& Vila, I. (2014). Estrategias discursivas y aprendizaje de la lengua escolar: un estudio etnográfico en un aula de alta diversidad étnica y lingüística. Cultura y Educación, 26(2), 349-376. https://doi.org/10.1080/11356405.2014.935112

Soriano, E. \& González, A. J. (2013). Las competencias emocionales en el ámbito afectivo-sexual de los adolescentes autóctonos e inmigrantes. Revista de Investigación Educativa, 31 (1), 133-149. doi:10.6018/rie.31.1.150531 https://doi.org/10.6018/rie.31.1.150531

SPSS Inc. Released 2011. IBM SPSS Statistics for Windows, Version 20.0. Armonk, NY: IBM Corp. 
